\title{
Noninvasive in-vivo optical properties of skin tumors
}

\section{Alejandro Garcia-Uribe, Elizabeth B. Smith, Jun Zou, Madeleine Duvic, Lihong V. Wang}

Alejandro Garcia-Uribe, Elizabeth B. Smith, Jun Zou, Madeleine Duvic, Lihong V. Wang, "Noninvasive in-vivo optical properties of skin tumors," Proc. SPIE 7175, Optical Interactions with Tissue and Cells XX, 717506 (24 February 2009); doi: 10.1117/12.808397

SPIE. Event: SPIE BiOS, 2009, San Jose, California, United States 


\title{
Noninvasive In-Vivo Optical Properties of Skin Tumors
}

\author{
Alejandro Garcia-Uribe ${ }^{1}$, Elizabeth B. Smith ${ }^{2}$, Jun Zou ${ }^{1}$, Madeleine Duvic ${ }^{3}$, Lihong V. Wang ${ }^{4}$ \\ ${ }^{1}$ Department of Electrical Engineering, Texas A\&M University, \\ 3128 TAMU, College Station, Texas, USA \\ ${ }^{2}$ Department of Biomedical Engineering, Texas A\&M University, \\ 3120 TAMU, College Station, Texas, USA \\ ${ }^{3}$ Department of Dermatology, the University of Texas MD Anderson Cancer Center, \\ 1515 Holcombe Blvd., Houston, Texas, USA \\ ${ }^{4}$ Department of Biomedical Engineering, Washington University in St. Louis, \\ One Brookings Drive, St. Louis, Missouri, USA
}

\begin{abstract}
This paper presents a study for in-vivo estimation of optical properties of pigmented skin tumor by oblique incidence diffuse reflectance spectroscopy. The developed system has been tested in clinical conditions to compare the optical properties of melanomas, dysplastic nevi and common nevi. The spatio-spectral data are collected in the wavelength range of 455 to $765 \mathrm{~nm}$ from 96 pigmented skin lesions including 10 histopathologically diagnosed as melanoma, 67 as dysplastic nevi and 19 lesions as common nevi. The preliminary results indicate significantly larger average reduced scattering coefficient spectra for malignant and dysplastic lesions than for benign common nevi.
\end{abstract}

Keywords: Absorption coefficient, scattering coefficient, spectroscopy, skin cancer, melanoma.

\section{INTRODUCTION}

Skin cancer is the most common form of cancer, with about a million new cases estimated in the U.S. each year. ${ }^{1}$ Melanoma is the most dangerous type of skin cancer and is the leading cause of death among the skin diseases. The American Cancer Society estimates that there are more than 62,000 new cases of melanoma in the United States every year. About 8,000 people are expected to die of this disease each year. Several researchers have studied the potential use of optical spectroscopy for early detection of cutaneous malignant tumors. ${ }^{2-6}$ Early detection and treatment of skin cancer can significantly improve patient outcomes. Our previous studies have shown that Oblique Incidence Diffuse Reflectance Spectrometry (OIDRS) as an effective tool to separate carcinoma skin cancer lesions from benign keratoses. $^{7}$ In clinical practice, dermatologists use the $\mathrm{ABCD}$ rule (asymmetry, border, color and diameter) and change in the appearance of a mole or pigmented area, to spot suspicious skin lesions. After the skin check a skin biopsy is preformed for assessing whether the pigmented lesion is benign or malignant. For early stage melanoma the treatment is usually a surgery to remove the tumor and a small amount of normal tissue around it. Dysplastic nevi are atypical moles with architectural disorder. Depending of the degree of atypia, dysplastic nevi are mild moderate or severe. Researchers believe that dysplastic nevi are more likely than ordinary moles to develop into a melanoma. ${ }^{8}$ For this reason severe dysplastic nevi are commonly surgically removed in the same way than melanoma.

\section{EXPERIMENTAL SETUP}

The design of the OIRDS probe consists of three source fibers and two linear arrays of twelve collection fibers within an area $2 \times 2 \mathrm{~mm}^{2}$ (Fig. 1). The small effective area can provide the needed spatial resolution for the detection of earlystage cancer lesions. Three micro-machined positioning devices are fabricated for accurate placement of the source and collection fibers. The position device for the source fibers consists of silicon substrate with lithographically patterned SU-8 guiding structures (100 $\mu \mathrm{m}$ thick). Each position device for the collection fibers consist of bulk etched channels on a silicon substrate. ${ }^{9}$ After assembling both source and collection fibers into the guiding structures, all the positioning chips are stacked and glued together with epoxy. The probe is then assembled into an aluminum holder to facilitate its clinical use.

Optical Interactions with Tissue and Cells XX, edited by Steven L. Jacques, E. Duco Jansen, William P. Roach,

Proc. of SPIE Vol. 7175, 717506 - @ 2009 SPIE · CCC code: 1605-7422/09/\$18 · doi: 10.1117/12.808397 


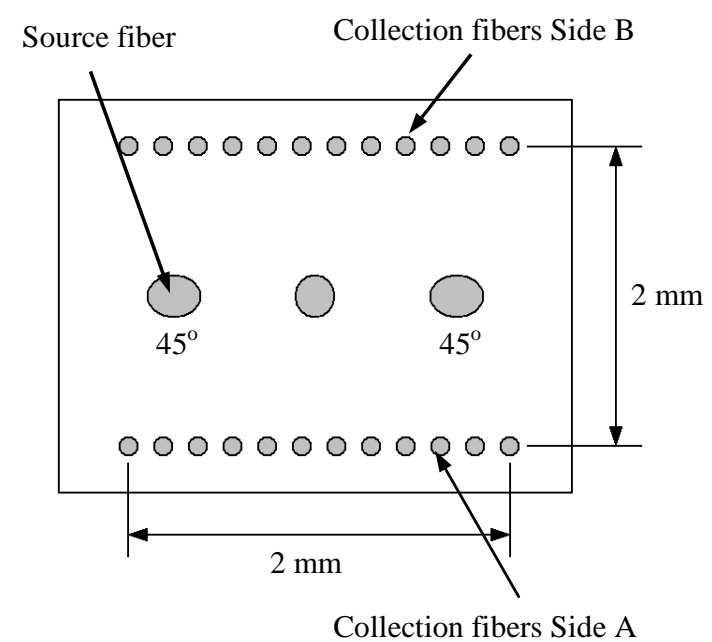

Fig. 1. Probe description.

The schematic of the experimental setup is illustrated in Fig. 2. White light from a halogen lamp is coupled to an optical fiber $(200 \mu \mathrm{m}$ diameter). The probe was placed gently on the skin area of interest without significant compression. An optical multiplexer allows delivering light through only one source fiber at a time to the area of interest. Once the light is delivered to the skin, it interacts with the medium and the diffuse reflectance is collected by another set of optical fibers. The collection fibers are coupled with an imaging spectrograph that generates an optical spectrum for each fiber. A CCD camera collects the spectral-images from the wavelength range 455 to $765 \mathrm{~nm}$. This information is stored on a computer for future data analysis.

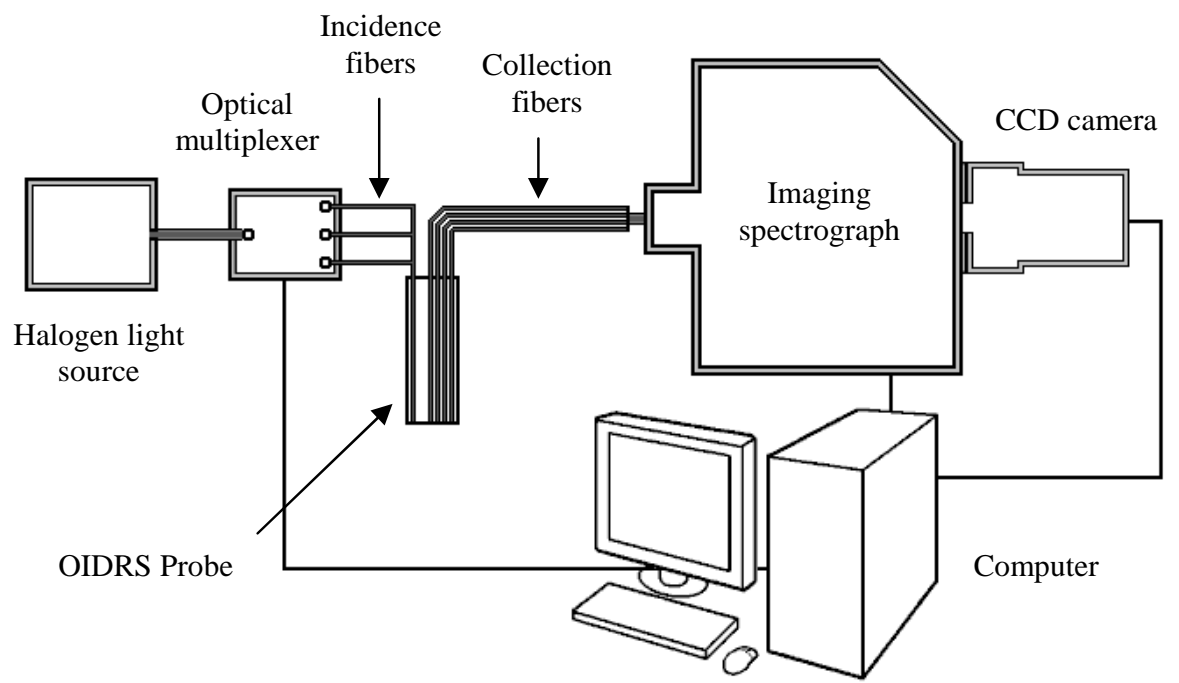

Fig. 2. System setup.

\section{OPTICAL PROPERTIES}

The spatially resolved steady-state diffuse reflectance for oblique incidence can be calculated using a modified twosource diffusion theory approximation with one positive source located below the sample surface and one negative 
located above the sample surface. ${ }^{10,11}$ The resolved steady-state diffuse reflectance a particular wavelength is described by

$$
R(x)=\frac{1}{4 \pi}\left[\frac{\Delta z\left(1+\mu_{e f f} \rho_{1}\right) \exp \left(-\mu_{e f f} \rho_{1}\right)}{\rho_{1}^{3}}+\frac{\left(\Delta z+2 z_{b}\right)\left(1+\mu_{e f f} \rho_{2}\right) \exp \left(-\mu_{e f f} \rho_{2}\right)}{\rho_{2}^{3}}\right]
$$

where $\rho_{1}$ and $\rho_{2}$ are the distances between the point of observation on the sample surface and the point of incidence. $\Delta \mathrm{z}$ is the distance between the virtual boundary and the tissue depth and $\mathrm{z}_{\mathrm{b}}$ is the distance between the virtual boundary and the sample surface. The effective attenuation coefficient $\left(\mu_{\text {eff }}\right)$ is defined by

$$
\mu_{\text {eff }}=\sqrt{\frac{\mu_{a}}{D}}
$$

where $D$ is the diffusion coefficient, $\mu_{a}$ is the absorption coefficient and $\mu_{s}^{\prime}$ is the reduced scattering coefficient. The diffusion coefficient can be calculated by

$$
D=\frac{1}{3\left(0.35 \mu_{a}+\mu_{s}^{\prime}\right)}
$$

The distance from the point of incidence to the positive point source $d_{s}=3 D$. The shift of the point sources in the $x$ direction $(\Delta x)$ is

$$
\Delta x=d_{s} \sin \left(\alpha_{t}\right)=\frac{\sin \left(\alpha_{t}\right)}{0.35 \mu_{a}+\mu_{s}{ }^{\prime}}
$$

where $\alpha_{\mathrm{t}}$ is the angle of refraction (Fig. 3). Using simple transformations the absorption and reduced scattering coefficients can be calculated by

$$
\begin{gathered}
\mu_{a}=\frac{\mu_{e f f}^{2} \Delta x}{3 \sin \left(\alpha_{t}\right)} \\
\mu_{s}^{\prime}=\frac{\sin \left(\alpha_{t}\right)}{\Delta x}-0.35 \mu_{a}
\end{gathered}
$$

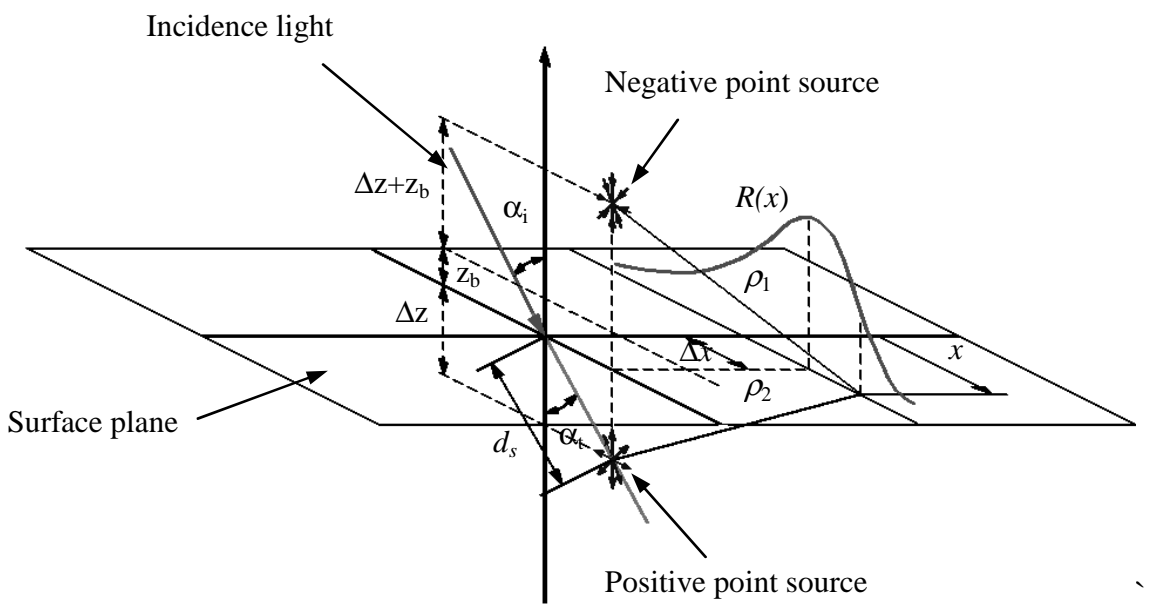

Fig 3. Oblique incidence diffuse reflectance for a single wavelength.

The OIDRS system was calibrated and validated using liquid phantoms made with polystyrene micro-spheres as scattering elements and trypan blue as absorber. 


\section{EXPERIMENTAL RESULTS}

The data acquisition from skin abnormalities was performed at The University of Texas M.D. Anderson Cancer Center (Melanoma and Skin Center). The OIRDS probe was used to collect spatio-spectral images from skin lesions and adjacent healthy skin. The diffuse reflectance spectra were collected from 96 pigmented skin lesion including 10 histopathologically diagnosed as malignant melanoma. Each spectral image from a lesion was normalized by a spectral image collected form the surrounding healthy tissue. An example of a spectral image is shown in Fig. 4.

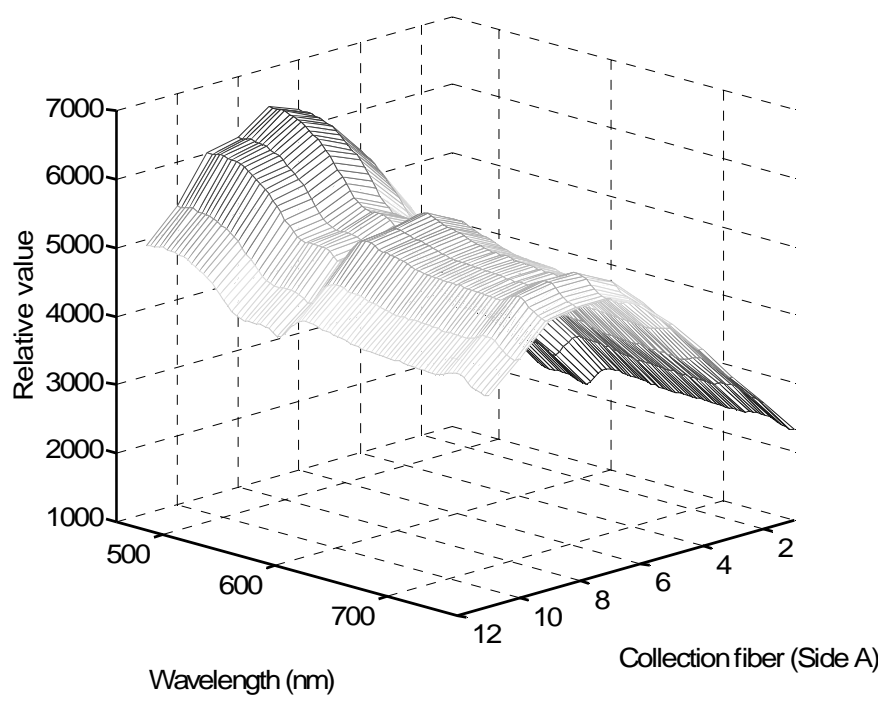

Fig. 4. In-vivo spatially resolved diffused reflectance.

The biopsy determined that the pigmented lesions in data set consists of benign common nevi (CN), mildly dysplastic nevi (DN1), moderately dysplastic nevi (DN2), severely dysplastic nevi (DN3), melanoma (M), The number of lesions is described in Table 1.

Table 1. Data description.

\begin{tabular}{|l|l|}
\hline Melanoma & 10 \\
\hline Severe Dysplastic Nevi & 5 \\
\hline Mild Dysplastic Nevi & 21 \\
\hline Moderate Dysplastic Nevi & 41 \\
\hline Common Nevi & 19 \\
\hline
\end{tabular}

After the measurements were completed, a biopsy was performed for each sample and submitted for histopathological diagnosis. The biopsy determined that the pigmented lesions in the data set consists of 10 melanomas, 67 dysplastic nevi and 19 common nevi. The scattering and absorption coefficient were estimated in for each skin lesion. The average scattering properties for malignant and dysplastic cases is larger than for the benign common nevi. The size of the scattering centers in cells is on the order of 100-1000's of nm, which is very similar to the wavelengths of light used in thr OIDRS system. Mitochondria, other cytoplasmic organelles and structures within the cell nuclei, are expected to be significant light scatterers and the average effective size of the scattering centers is on the order of a few hundred $\mathrm{nm} .{ }^{12,13}$ The absorption coefficient spectra for melanoma and dysplastic cases are generally higher than for the benign ones. The melanoma cases have similar average absorption spectra with that of the dysplastic cases. 


\section{SUMMARY AND DISCUSSION}

The presented system can estimate the in-vivo optical properties of skin tumors. This information can potentially be used to assist the photodynamic therapy, and in-vivo and non-invasive diagnosis of human skin pathologies. This higher scattering coefficient for the melanoma cases could be explained by larger average effective size of the scattering centers. Melanoma has a higher absorption coefficient for the melanoma cases can be related to higher volume-averaged concentration of hemoglobin, which could be indicative of angiogenesis cancerous lesions.

\section{ACKNOWLEDGMENTS}

The authors thank Dr. Mays, Dr. Hymens and Dr. Mansfield and the staff from the "Melanoma and Skin Center" at the University of Texas M. D. Anderson Cancer Center for their help during the data collection. This project is sponsored by National Institute of Health grant R01 CA106728.

\section{REFERENCES}

1. American Cancer Society website, "Overview: skin cancer - Melanoma” Available: http://www.cancer.org.

2. V. P. Wallace, J. C. Bamber, D. C. Crawford, R. J. Ott, and P. S. Mortimer, "Classification of reflectance spectra from pigmented skin lesions, a comparison of multivariate discriminant analysis and artificial neural networks," Phys. Med. Biol. 45, 2859-2871 (2000).

3. L. M. McIntosh, R. Summers, M. Jackson, H. H. Mantsch, J. R. Mansfield, M. Howlett, A. N. Crowson, J. W. Toole, “Towards non-invasive screening of skin lesions by near-infrared spectroscopy," Journal of Investigative Dermatology 116, 175-181 (2001).

4. S. Tomatis, M. Carrra, A. Bono, C. Bartoli, M. Lualdi, G. Tragni, A. Colombo, R. Marchesini, "Automated melanoma detection with a novel multispectral imaging system: results of a prospective study," Phys. Med. Biol. 50, 1675-1687 (2005).

5. D. Arifler, R. A. Schwarz, S. K. Chang, R. Richards-Kortum. "Reflectance Spectroscopy For Diagnosis Of Epithelial Precancer: Mmodel-Based Analysis of Fiber-Optic Probe Designs to Resolve Spectral Information From Epithelium And Stroma," Appl. Opt. 44, 4291-4305 (2005).

6. S. Sigurdsson, P. A. Philipsen, L. K. Hansen, J. Larsen, M. Gniadecka, and H. C. Wulf, "Detection of skin cancer by classification of raman spectra", IEEE Transactions on Biomedical Engineering 51, 1784-1793 (2004).

7. A. Garcia-Uribe, N. Kehtarnavaz, G. Marquez, V. Prieto, M. Duvic, and L. V. Wang, "Skin Cancer Detection by Spectroscopic Oblique-Incidence Reflectometry: Classification and Physiological Origins," Appl. Opt. 43, 2643-2650 (2004)

8. M. L. Williams and R. W. Sagebiel, "Melanoma risk factors and atypical moles," West J Med. 160, 343-350 (1994).

9. A. Garcia-Uribe, K. C. Balareddy, J. Zou and L. V. Wang, "Micromachined Fiber Optical Sensor for In-Vivo Measurement of Optical Properties of Human Skin," IEEE Sensors Journal 8, 1698-1703 (2008).

10. L. H. Wang and S. L. Jacques, "Use of a laser beam with an oblique angle of incidence to measure the reduced scattering coefficient of a turbid medium," Appl. Opt. 34, 2362-2366 (1995).

11. S. -P. Lin, L. Wang, S. L. Jacques, and F. K. Tittel, "Measurement of tissue optical properties by the use of oblique-incidence optical fiber reflectometry," Appl. Opt. 36, 136-143 (1997)

12. J. R. Mourant, J. P. Freyer, A. H. Hielscher, A. A. Eick, D. Shen, and T. M. Johnson, "Mechanisms of light scattering from biological cells relevant to noninvasive optical-tissue diagnostics," Appl. Opt. 37, 3586-3593 (1998).

13. J. R. Mourant, M. Canpolat, C. Brocker, O. Esponda-Ramos, T. M. Johnson, A. Matanock, K. Stetter, and J. P. Freyer, "Light scattering from cells: the contribution of the nucleus and the effects of proliferative status," J. Biomed. Opt. 5, 131-137 (2000). 